\title{
Physikalische und numerische Modellierung des Strömungsverhaltens im Stauraum des KW Feistritz- Ludmannsdorf an der Drau
}

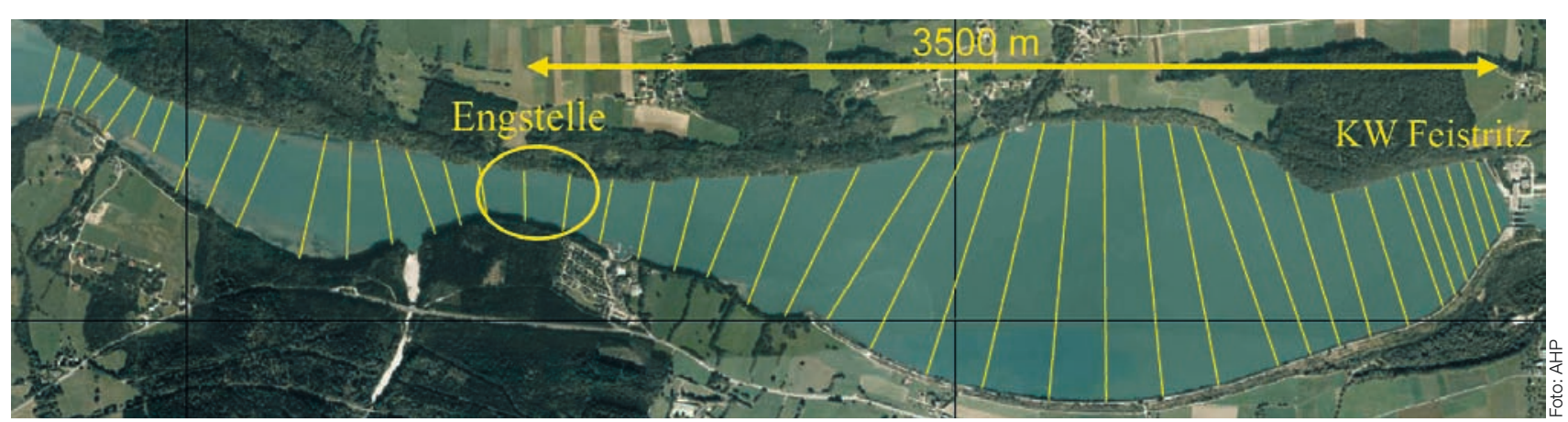

Abb. 1: Lageplan Stauraum KW Feistritz-Ludmannsdorf, Projektgebiet.

Zusammenfassung: Die Aufweitung im Stauraum des Kraftwerks Feistritz-Ludmannsdorf an der Drau verursacht im breiten Bereich des Stauraums in Abhängigkeit von Wasserführung und Wasserspiegelhöhe ein stark wechselndes Strömungsverhalten. Diese Strömungsumlagerungen bewirken eine ungeordnete Schwebstoffverlandung. Ein zukunftsorientiertes Konzept sieht die Errichtung von Leitwerken vor, mittels derer für alle hydraulischen Randbedingungen dieselbe Strömungsverteilung erreicht und damit die ungeordnete Schwebstoffverlandung im Stauraum verhindert wird. Für dieses Vorhaben wurden numerische Modelle und ein darauf aufbauender physikalischer Modellversuch im neuen Labor des Instituts für Wasserbau und Wasserwirtschaft, Technische Universität Graz, eingesetzt. Numerisches und physikalisches Modell wurden mit räumlich hochauflösenden ADCP Naturmessungen kalibriert. Der Vergleich des Strömungsverhaltens zwischen Naturmessung, Numerik und Modellversuch zeigt für unterschiedliche hydraulische Randbedingungen sehr gut übereinstimmende Ergebnisse.

Physical and numerical modelling of flow behaviour in the reservoir above the Feistritz-Ludmannsdorf power station on the River Drau in Carinthia

Institut für Wasserbau und Wasserwirtschaft, Technische Universität Graz

2 Verbund-Austrian Hydro Power AG, Wien
Summary: Since the reservoir above the Feistritz-Ludmannsdorf power station on the River Drau was widened, flow behaviour in the wide zone of the reservoir has been seen to vary considerably as a function of flow rate and water surface level. This changing flow pattern in turn causes erratic deposition of suspended sediment. A future-oriented project concept involves the provision of training works intended to ensure a constant flow distribution for all hydraulic conditions so as to prevent the irregular sedimentation of suspended matter. Numerical models and a model test based on them have been used at the new laboratory of the Department of Hydraulic Engineering and Water Resources Management of the Graz University of Technology to study this problem. The numerical and physical models were calibrated using high-spatial-resolution ADCP field measure- ments. Comparison of flow behaviour as measured in the field and obtained by numerical and experimental means shows very good agreement for different hydraulic conditions.

\section{Einleitung}

Das Wasserkraftwerk Feistritz-Ludmannsdorf ist Teil der österreichischen Kraftwerkskette an der Drau, zu der neun weitere Anlagen gehören. Es wurde 1968 in Betrieb genommen, die Engpassleistung beträgt $88 \mathrm{MW}$. Durch den Aufstau entstand ein ca. $15 \mathrm{~km}$ langer und bis zu $1000 \mathrm{~m}$ breiter Stausee mit Wassertiefen von bis zu $20 \mathrm{~m}$. Bei einem jährlichen Feststofftransport in der Drau von ca. 1 Mio. $\mathrm{m}^{3}$ sedimentieren davon ca. $90 \%$ in den Stauräumen der mittleren Drau. Die zumindest einmal pro Jahr durchge-

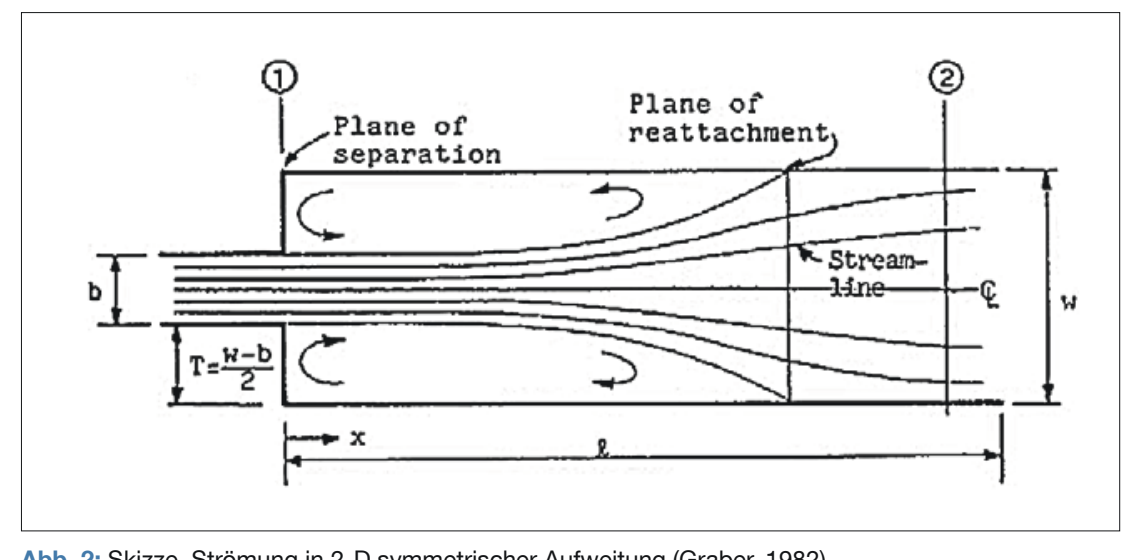

Abb. 2: Skizze, Strömung in 2-D symmetrischer Aufweitung (Graber, 1982). 


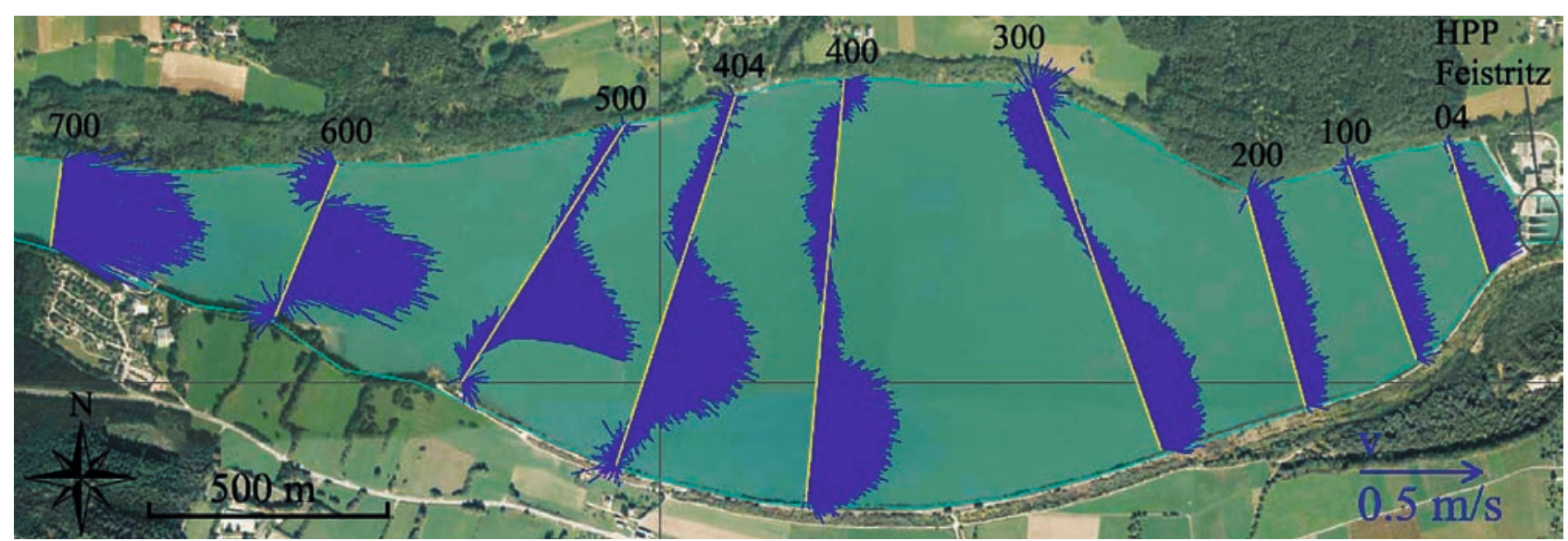

Abb. 3: Lageplan, tiefengemittelte ADCP Fließgeschwindigkeiten.

führten Profilaufnahmen im Stauraum des KW Feistritz-Ludmannsdorf zeigen die Ausbildung von ausgedehnten Ablagerungsfeldern. Als bisherige Gegenmaßnahme wurden jährliche Baggerungen von ca. $150.000 \mathrm{~m}^{3}$ im Stauraum zur Aufrecherhaltung der Hochwassersicherheit durchgeführt. Im Jahr 2007 wurden im Projektgebiet (Abb. 1) zusätzlich zu den 14 jährlich gemessenen Querprofilen weitere 29 Profile mittels Echolot vermessen. Die Überlagerung der Sohlaufnahmen für das Projektgebiet aus den Jahren 1968 (Ausgangszustand) und 2007 zeigt, dass in diesem Zeitraum der durchschnittliche Feststoffeintrag ca. $400.000 \mathrm{~m}^{3}$ pro Jahr betrug. Die durchschnittliche Verlandungshöhe im Stauraum beträgt 4,60 m. Das ursprüngliche Flussbett der Drau war am orographisch rechten Ufer situiert, die Verlandungshöhen betragen dort bis zu $15 \mathrm{~m}$.

Eine zukunftsorientierte Lösung sieht die Schüttung von Leitwerken vor, mittels derer eine Tiefenrinne für die Sedimenttrift im Bereich bis ca. $3500 \mathrm{~m}$ oberhalb des Wehres gewährleistet werden soll. Dadurch soll eine optimale Durchschleusung der Schwebstoffe und mobilisierten Sedimente bei Stauabsenkungen sowie die Minimierung der Sedimentation im Normalbetrieb bewirkt werden. Die genaue Kenntnis der Geschwindigkeitsverteilung bei verschiedenen Wasserführungen hat einen entscheidenden Einfluss auf die Lage und Anordnung der Leitwerke. Um eine möglichst effiziente Wirkung der Leitwerke in der Natur zu erzielen, werden numerische Berechnungen und zur Validierung ein damit in Verbindung stehender physikalischer Modellversuch (M 1:100 horizontal/M 1:25 vertikal) durchgeführt.

\subsection{Dreidimensionales} Aufweitungsproblem

Das Projektgebiet im Stauraum ist gekennzeichnet von einer $150 \mathrm{~m}$ breiten Engstelle 3500 flussauf vom Wehr (Abb. 1). Flussab dieser Engstelle weitet sich der Stauraum bis zu einer Breite von $1000 \mathrm{~m}$ auf und die Wassertiefe steigt von ca. $5 \mathrm{~m}$ auf 15-20 m an. Diese Situation stellt eine dreidimensionale Aufweitung dar.

In Anlehnung an zahlreiche Literatur über Strömungsverhalten in plötzlichen Querschnittserweiterungen, z.B. Graber (1982), können schon geringe geometrische Abweichungen am Beginn der Aufweitung eine bedeutende Strömungsablenkung verursachen. Im Gegensatz zu den skizzierten Strömungslinien in Abbildung 2 würde sich folglich auf einer Strecke L eine über den Querschnitt ungleich- förmige Strömungsverteilung einstellen.

Bezugnehmend auf das Projektgebiet bedeutet dieser Sachverhalt, dass die Strömung im breiten Bereich des Stauraums sehr sensibel auf die im Bereich der Engstelle herrschenden topographischen und hydraulischen Randbedingungen reagiert. Diese Problematik verdeutlicht, dass gerade in diesem Fall eine Kalibrierung des numerischen Modells und auch des physikalischen Modellversuchs durch Naturmessungen unerlässlich ist.

\section{ADCP Geschwindigkeits- messungen im Stauraum}

Für die Ermittlung der Geschwindigkeitsverteilung im Projektgebiet wurde ein ADCP (Acoustic Doppler Current Profiler) WorkHorse ${ }^{\circledR}$ RioGrande $1200 \mathrm{kHz}$ der Firma RD Instruments verwendet. Das

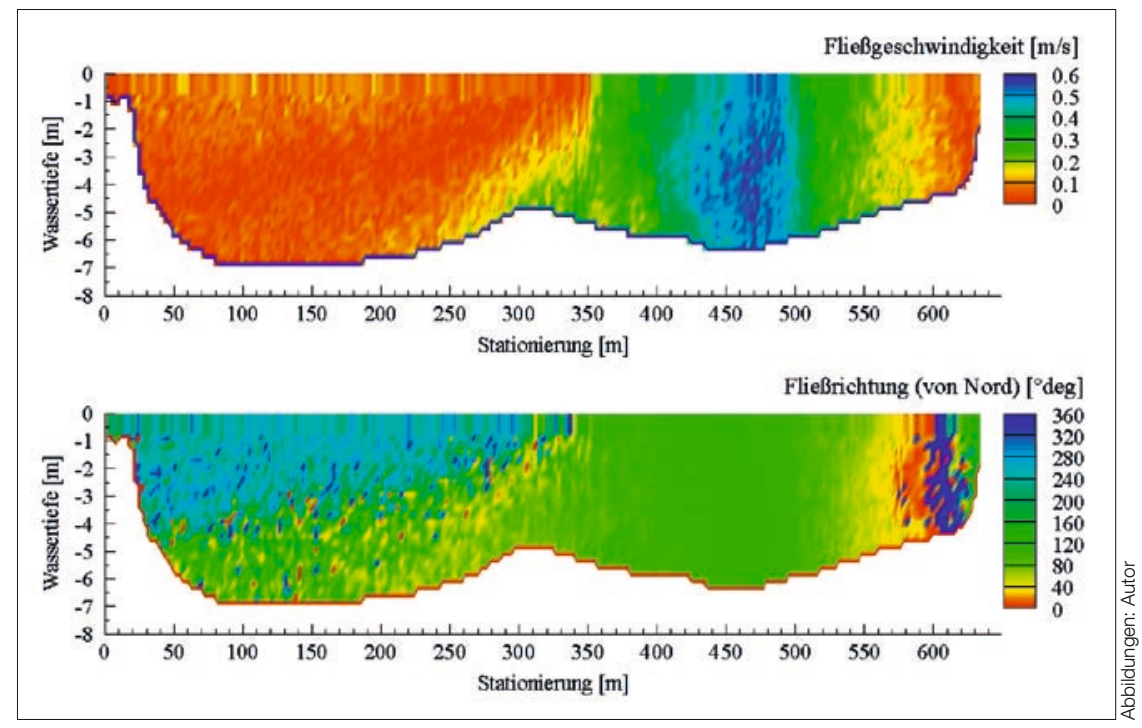

Abb. 4: Querschnitt 500, ADCP Fließgeschwindigkeiten (oben), Fließrichtungen (unten). 
Messprinzip dieses Geräts beruht auf dem Dopplereffekt, Ultraschallsignale werden mit einer bestimmten Frequenz ausgesendet, von kleinsten Teilchen im Wasser reflektiert und wiederum empfangen. Die Annahme ist dabei, dass sich diese Teilchen mit der gleichen Geschwindigkeit wie das Wasser bewegen (RD Instruments, 1996). Das ADCP Gerät misst in verschiedenen Wassertiefen die Fließgeschwindigkeiten in allen drei Raumrichtungen. Bei der Moving-Boat Messung wird mittels integriertem Kompass und Wassertiefenmessung die Bootsgeschwindigkeit kontinuierlich aufgezeichnet. Die tatsächlichen Fließgeschwindigkeiten ergeben sich aus der Subtraktion der vektoriellen Bootsgeschwindigkeiten von den Rohmessdaten.

Insgesamt wurden 15 Querprofile mit dem Messboot aufgenommen, wobei die kontinuierliche Position über ein differentielles GPS bestimmt wurde. Vom Kraftwerksbetreiber wurden ein konstanter Turbinendurchfluss $\mathrm{Q}=440 \mathrm{~m}^{3} / \mathrm{s}$ und ein konstanter Stauspiegel STZ $=461,35$ müA zur Verfügung gestellt, um für die Messphase einen stationären Strömungszustand im Stauraum zu gewährleisten. In Abbildung 3 sind für den Bereich flussab der Engstelle die Messergebnisse als tiefengemittelte Fließgeschwindigkeiten dargestellt.

Flussab der Engstelle (Querschnitt 700) bildet sich die Hauptströmung am orographisch rechten Ufer aus, gleichzeitig zei- gen sich ausgedehnte Rückströmungen und Stillwasserzonen am orographisch linken Ufer. Aufgrund der geringen Wassertiefe am orographisch rechten Ufer zwischen den Querschnitten 600 und 500 sind in diesem Bereich auch Ablösezonen zu verzeichnen. Flussab des Querprofils 200 sind die Fließgeschwindigkeiten annähernd gleichförmig über den Querschnitt verteilt.

In Abbildung 4 sind die Fließgeschwindigkeiten (oben) und die Fließrichtungen (unten) im Querschnitt 500 dargestellt. Die Analyse der Fließrichtungen zeigt einen vertikalen Wirbel im orographisch linken Bereich; bis zu einer Wassertiefe von $4 \mathrm{~m}$ bewegt sich die Strömung flussauf, in Sohlnähe hingegen bewegt sich die Strömung flussab.

Die ADCP Messungen zeigen das komplexe Strömungsverhalten im Stauraum und dessen dreidimensionalen Charakter. Die hydraulischen Randbedingungen (Messbedingungen) im Stauraum verursachen eine hohe gerätespezifische Standardabweichung und damit Ungenauigkeit der Messergebnisse, jedoch können die Hauptströmungsrichtung, Rückströmungen und Stillwasserzonen detailliert aufgelöst werden.

\section{3-D numerisches Modell}

Die in diesem Beitrag vorgestellten numerischen Ergebnisse wurden mit dem

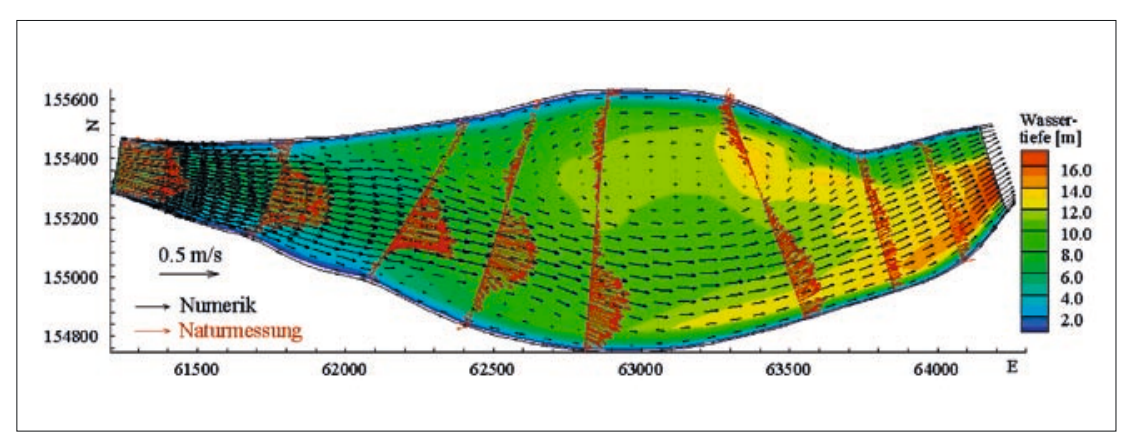

Abb. 5: Lageplan, tiefengemittelte Geschwindigkeiten: ADCP Naturmessung - Numerik.
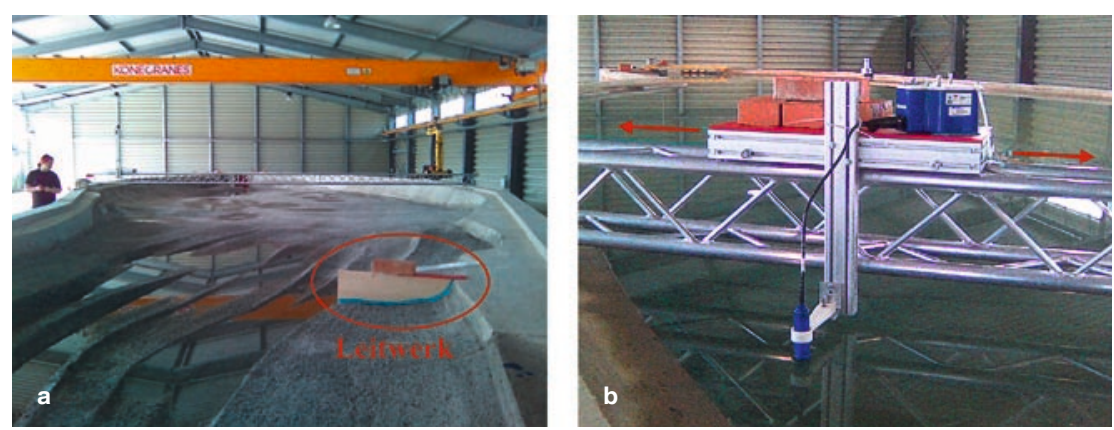

Abb. 6: a) Modellversuch, Blick in Fließrichtung, b) ADCP StreamPro Sonde.
Finite Volumen Code SSIIM (Olsen, 2007) durchgeführt. Die Reynolds gemittelten Navier-Stokes Gleichungen wurden an einem unstrukturierten, nicht versetzten Netz gelöst. Für die Turbulenzschließung wurde das Standard k- $\varepsilon$ Modell verwendet. Für die Bettzelle wurde das logarithmische Wandgesetz für eine raue Wand benutzt.

Das 3-D numerische Modell wurde mit den im vorigen Kapitel beschriebenen ADCP Naturmessungen kalibriert. Die Kalibrierung beschränkte sich auf einen zweidimensionalen Vergleich der tiefengemittelten Fließgeschwindigkeiten. Das Modellgebiet konnte aufgrund der Kenntnis der Strömungsverhältnisse in der Engstelle auf den Bereich zwischen Engstelle und Wehr reduziert werden. Der Einströmwinkel an der oberen Randbedingung wurde gleich dem dort gemessenen mittleren Fließwinkel aus der ADCP Naturmessung gesetzt. Mit der Wahl einer äquivalenten Sandrauigkeit von $\mathrm{k}_{\mathrm{s}}=0,02 \mathrm{~m}$ (entspricht ca. 100 $\mathrm{d}_{90}$ ) für das gesamte Modellgebiet konnte eine sehr gute Übereinstimmung mit den Naturmessungen erreicht werden ( $A b b .5)$.

Der Vergleich der Ergebnisse für die Fließgeschwindigkeiten ( $A b b$. 5) zeigt, dass das numerische Modell die große Rückströmzone auf der orographisch linken Seite auflöst und auch die Ablösezone am orographisch rechten Ufer reproduziert. Während die Fließgeschwindigkeiten am Einlass und Auslass sehr gut übereinstimmen, sind im breiten Bereich des Stauraums die Kernzonen hoher Geschwindigkeiten in der Natur im Vergleich zur Numerik wesentlich kompakter. Gründe dafür können sein: a) das k- $\varepsilon$ Turbulenzmodell überschätzt generell die Wirbelviskosität und dämpft damit die Strömung, b) Rauigkeitsunterschiede im Stauraum, c) falsche Diffusion verschmiert die Ablösezonen. Einige Parametervariationen diesbezüglich werden in Dorfmann und Knoblauch (2009) präsentiert, wo unter anderem der bedeutende Einfluss der gewählten Rauigkeit und des verwendeten Differenzierungsschemas auf das Strömungsverhalten diskutiert wird.

Die weiteren Berechnungen aufGrundlage des kalibrierten numerischen Modells zeigten, dass sich bei einer Absenkung des Stauspiegels um 2,50 m der Hauptstromstrich zur orographisch linken Seite verlagert. Dieser Lastfall wurde bei einem kleineren Hochwasserereignis (ca. $\mathrm{HQ}_{1}$ ) beobachtet und in weiterer Folge im physikalischen Modellversuch bestätigt. Im numerischen Modell wurden Leit- 


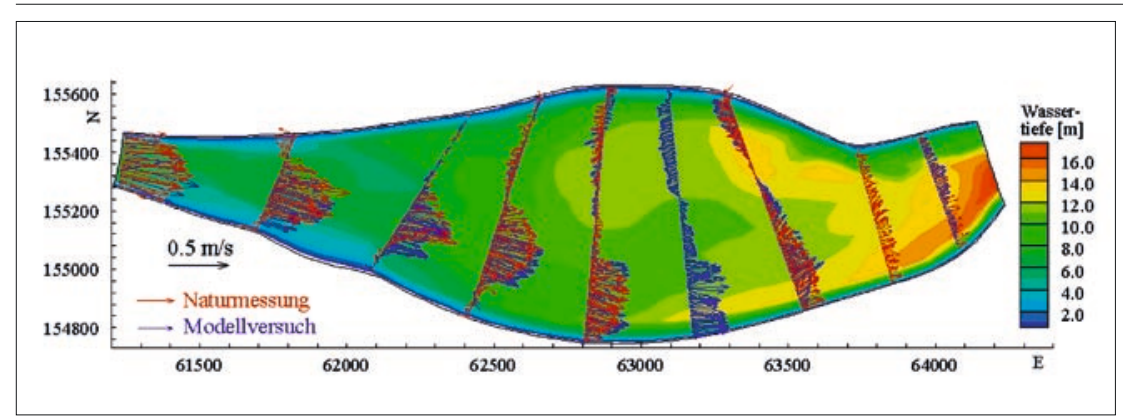

Abb. 7: Turbinenbetrieb, Fließgeschwindigkeiten: Modellversuch - Naturmessung.

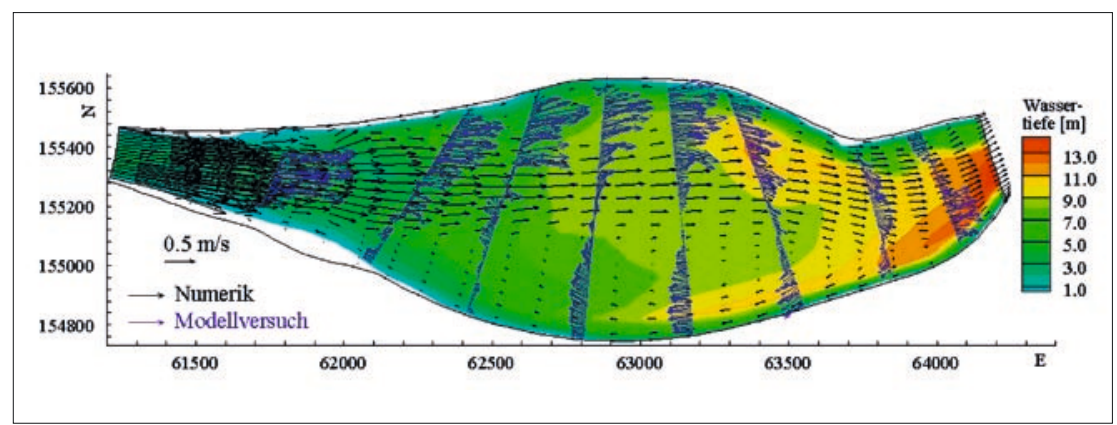

Abb. 8: Wehrbetrieb, Fließgeschwindigkeiten: Modellversuch - Numerik.

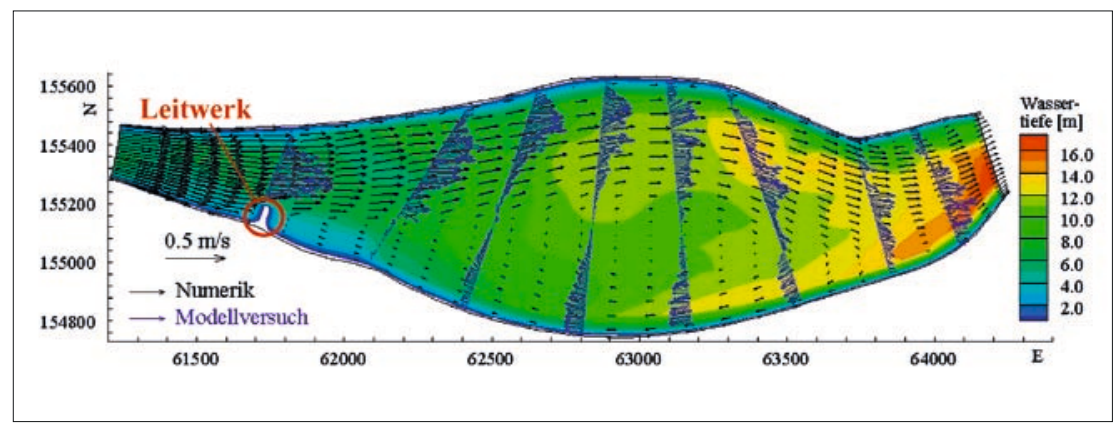

Abb. 9: Turbinenbetrieb mit Einbau eines Leitwerks, Fließgeschwindigkeiten: Modellversuch - Numerik.

werke mit dem primären Ziel eingebaut, den Hauptstromstrich für verschiedene Lastfälle lagemäßig beizubehalten und damit keine ungeordnete Schwebstoffverlandung zu bewirken. Das zweite Ziel war wirtschaftlicher Natur - die Leitwerke sollten bei einem möglichst geringen baulichen Aufwand die größtmögliche Effizienz aufweisen.

\section{Physikalischer Modellversuch}

Der physikalische Modellversuch wurde im neuen Wasserbaulabor des Instituts für Wasserbau und Wasserwirtschaft der TU Graz nach dem Froude'schen Modellgesetz durchgeführt. Die beachtliche Größe des zu modellierenden Stauraumabschnitts bedingte einen kleinen Modellmaßstab für die horizontalen Längen von M 1:100. 2-D numerische Voruntersuchungen zeigten, dass sich bei diesem Maßstab in weiten Bereichen des Modell-
Beschreibung der Strömungsverhältnisse wären konventionelle Punktmessungen, z.B. mit ADV Vectrino, aufgrund der Größe des Modells sehr zeitaufwendig gewesen. Aus diesem Grund wurde erstmals in einem physikalischen Modellversuch eine ADCP StreamPro Sonde der Firma RD Instruments eingesetzt, welche ursprünglich für Naturmessungen bei geringen Wassertiefen und Abflüssen entwickelt wurde. Das Messprinzip ist dasselbe wie bei den größeren ADCP RioGrande Sonden. Die Sonde wurde mittels eines Motors mit einer mittleren Geschwindigkeit von $1 \mathrm{~cm} / \mathrm{s}$ entlang der Querprofile geführt (Abb. 6b). Im Modellversuch wurden nur mehr jene Leitwerkvarianten getestet und überprüft, mit welchen in der zuvor durchgeführten numerischen Modellierung die geforderten Ziele erreicht wurden.

\section{Ergebnisse}

Im Folgenden werden ausgewählte Ergebnisse aus dem physikalischen Modellversuch der Naturmessung und den numerischen Berechnungen gegenübergestellt. Die Auswertung erfolgte über den Vergleich der tiefengemittelten Geschwindigkeiten.

Die ersten Ergebnisse bezogen sich auf die Kalibrierung der Strömungssituation zwischen Modell und Natur. Abbildung 7 zeigt den Vergleich zwischen Modellversuch und Naturmessung für stationären Turbinenbetrieb mit der Ausbauwassermenge $\mathrm{Q}=415-440 \mathrm{~m}^{3} / \mathrm{s}$ bei einem Wasserspiegel WSP $=461,35-461,50$ müA. Die Fließgeschwindigkeiten im Modellversuch stimmen lagemäßig und quantitativ sehr gut mit den in der Natur gemessenen überein. So können der dem Uhrzeigersinn entgegengesetzte Wirbel auf der linken Seite und auch die teils abrupten Ablösezonen auf der rechten Seite fast identisch reproduziert werden.

Das übereinstimmende Ergebnis weist zudem darauf hin, dass die Stauraumgeometrie im physikalischen Modell sehr gut nachgebaut wurde und unterstreicht, dass die ADCP Messungen in Natur und Modell zuverlässige Messdaten liefern.

Abbildung 8 zeigt das stationäre Strömungsverhalten im Modellversuch und in der numerischen Simulation bei einer Stauspiegelabsenkung von 2,50 $\mathrm{m}$ und einem Durchfluss von $Q=700 \mathrm{~m}^{3} / \mathrm{s}$ (dies entspricht einem $\mathrm{Q}$ von ca. $0,7 \mathrm{HQ}_{1}$ ). Sowohl im numerischen Modell als auch im Modellversuch wurden nur diese hydraulischen Randbedingungen geändert. Im 
physikalischen Modellversuch ergibt sich im Vergleich zum Lastfall Turbinenbetrieb eine komplette Strömungsumlagerung vom rechten zum linken Ufer. Im numerischen Modell verlagert sich die Strömung nur tendenziell an das orographisch linke Ufer. Die Strömungsumlagerung kann damit begründet werden, dass unmittelbar flussab der Engstelle bei einer Stauspiegelabsenkung am orographisch rechten Ufer ausgedehnte Flachwasserzonen trocken fallen. Diese wirken als natürliche Buhne, engen den Fließquerschnitt ein und der Strömung wird ein Impuls in Richtung orographisch linke Seite induziert. Der im numerischen Modell unterschätzte Umschlag der Strömung ist darauf zurückzuführen, dass gerade unmittelbar flussab der Engstelle die Stauraumgeometrie sehr unstetig ist und für diesen Bereich die digitale Geländemodellierung ungenauer erfolgte als der physikalische Modellnachbau.

Die wechselnden Strömungsverhältnisse begünstigen maßgeblich die ungeordnete Schwebstoffverlandung und minimieren die Entlandungswirkung bei Stauabsenkungen. Weitere ADCP Naturmessungen haben gezeigt, dass sich bei einer Absenkung des Wasserspiegels von 1,0 m unter das Stauziel der Hauptstromstrich genau in die Mitte verlagert. Dies deutet darauf hin, dass sich bereits bei normalem Schwellbetrieb (Stauspiegelabsenkung von max. $-0,5 \mathrm{~m}$ )eine zwischen rechtem Ufer und Stauraummitte pendelnde Hauptströmung einstellt.

\subsection{Einbau eines Leitwerks}

Primäre Funktion der Leitwerke ist, für alle hydraulischen Randbedingungen dieselbe Strömungsverteilung im breiten Bereich des Stauraums zu bewirken.

Eine in der numerischen Berechnung erfolgversprechende Leitwerkvariante war, für den Lastfall Turbinenbetrieb den Hauptstromstrich an das orographisch linke Ufer zu lenken und somit für alle hydraulischen Randbedingungen dasselbe Strömungsbild zu erzeugen. Durch zahlreiche Variantenrechnungen konnte das nicht überströmbare Leitwerk sukzessiv optimal positioniert und auf ca. $70 \mathrm{~m}$ Länge optimiert werden. Aufgrund des guten numerischen Ergebnisses wurde diese
Variante im physikalischen Modellversuch überprüft ( $A b b$. 6a). Die Ergebnisse für den Lastfall Turbinenbetrieb mit einem $70 \mathrm{~m}$ langen Leitwerk werden in $\mathrm{Ab}$ bildung 9 präsentiert. Im Vergleich zum Strömungsbild in Abbildung 7 bewirkt das Leitwerk eine völlige Strömungsumlagerung vom rechten zum linken Ufer. Die Gegenüberstellung von numerischer Simulation und Modellversuch zeigt ein sehr gut übereinstimmendes Ergebnis. Mit dem Einbau des Leitwerks wird die Strömung sowohl im numerischen als auch im physikalischen Modell stabilisiert. Das Leitwerk in dieser Position ist nur bei Stauziel aktiv, d. h. bei Stauspiegelabsenkungen fällt es teilweise bzw. ganz trocken. Dies hat den Vorteil, dass bei Hochwässern mit Stauspiegelabsenkung das Leitwerk keinem bzw. nur einem reduzierten Strömungsangriff ausgesetzt wird. Die im Vergleich zum physikalischen Modell geringeren Fließgeschwindigkeiten in der numerischen Berechnung sind auf die schon in Kapitel 3 genannten Gründe zurückzuführen.

In einer anderen Variante im numerischen Modell konnte durch die Anordnung von zwei Leitwerken ein mittiger Hauptstromstrich mit Rückströmungen auf der orographisch linken und rechten Seite erzeugt werden. Im Modellversuch konnte diese Variante jedoch nicht reproduziert werden. Vielmehr stellte sich ein instabiles, auf äußere Einflüsse (z. B. Sohländerungen) sensibel reagierendes Strömungsverhalten ein.

\section{Zusammenfassung}

Die Aufweitung im Stauraum des Kraftwerks Feistritz-Ludmannsdorf an der Drau verursacht im breiten Bereich des Stauraums in Abhängigkeit von Wasserführung und Wasserspiegelhöhe ein stark wechselndes Strömungsverhalten. Diese Strömungsumlagerungen bewirken eine ungeordnete Schwebstoffverlandung. Ein zukunftsorientiertes Konzept sieht die Errichtung von Leitwerken vor, mittels derer für alle hydraulischen Randbedingungen dieselbe Strömungsverteilung erreicht und damit die ungeordnete Schwebstoffverlandung verhindert wird. Für dieses Vorhaben wurden numerische Modelle und ein darauf aufbauender physikalischer Modellversuch im neuen Labor des Instituts für Wasserbau und Wasserwirtschaft, Technische Universität Graz, eingesetzt. Numerisches und physikalisches Modell wurden mit räumlich hochauflösenden ADCP Naturmessungen kalibriert. Durch die genaue Kenntnis des Status-quo-Strömungsverhaltens konnte unter anderem die in diesem Beitrag vorgestellte Leitwerkvariante hinsichtlich Effizienz aber auch Kostenaufwand ideal positioniert und deren Länge minimiert werden. Der Vergleich des Strömungsverhaltens zwischen Naturmessung, Numerik und Modellversuch zeigt für unterschiedliche hydraulische Randbedingungen sehr gut übereinstimmende Ergebnisse. Im physikalischen Modellversuch wurden nur mehr jene Leitwerkvarianten überprüft, welche sich in den numerischen Berechnungen als effizient erwiesen. Deshalb konnte die Versuchsdauer im Modellversuch wesentlich verkürzt werden.

\section{Danksagung}

Die Autoren bedanken sich bei der Verbund - Austrian Hydro Power AG (AHP) und dem Land Steiermark, der Abteilung Hydrographie, für die Unterstützung bei den ADCP Naturmessungen und den ADCP StreamPro Messungen im physikalischen Modellversuch.

Korrespondenz:

DI Clemens Dorfmann

Institut für Wasserbau und Wasserwirtschaft

Technische Universität Graz

Stremayrgasse 10/l

A-8010 Graz

E-Mail: clemens.dorfmann@tugraz.at

\section{LITERATUR}

Dorfmann C, Knoblauch H (2009) Calibration of 2-D and 3-D Numerical Models of a Large Reservoir Using ADCP Measurements, Proceedings of the 33rd IAHR Congress, Vancouver, Canada, pp. 1332-1339

Graber D (1984) Asymmetric Flow in Symmetric Expansions, Journal of the Hydraulics Divisions, ASCE, 108 (HY10), pp. 1082-1101

Olsen NRB (2007) SSIIM (http://folk.ntnu.no/nilsol/ssiim), The Norwegian University of Science and Technology NTNU, Trondheim, Norway RD Instruments (1996) Principles of Operation - A Practical Primer, 2nd Edition for Broadband ADCPs, San Diego, CA 